\title{
Detailed comparison of energy-related time-use diaries and monitored residential electricity demand
}

\author{
Kiti Suomalainen ${ }^{1 a}$, David Eyers ${ }^{\mathrm{c}}$, Rebecca Ford ${ }^{\mathrm{d}}$, Janet Stephenson ${ }^{\mathrm{a}}$, Ben \\ Anderson $^{\mathrm{e}}$, Michael Jack ${ }^{\mathrm{b}}$ \\ ${ }^{a}$ Centre for Sustainability, University of Otago, 563 Castle St., Dunedin 9054, New Zealand \\ ${ }^{b}$ Department of Physics, University of Otago, 730 Cumberland St., Dunedin 9016, New \\ Zealand \\ ${ }^{c}$ Department of Computer Science, University of Otago, 133 Union Street East, Dunedin \\ 9016, New Zealand \\ ${ }^{d}$ Environmental Change Institute, University of Oxford Centre for the Environment, South \\ Parks Road, Oxford, OX1 3QY, United Kingdom \\ ${ }^{e}$ Energy $\& 3$ Climate Change Division, Faculty of Engineering and the Environment, \\ University of Southampton, University Road, Southampton, SO17 1BJ, United Kingdon
}

\begin{abstract}
Understanding demand flexibility in the residential sector depends on understanding the causal link between household occupants' activities and resulting electricity demand. Self-reported electricity use via time-use diaries is often used as a direct descriptor of occupants' activities and has been integrated into residential electricity demand simulation models. Conversely, smart meter electricity demand data is increasingly used to infer occupants' activities. Underlying both these approaches are a number of unverified assumptions about people's perceptions of their energy use, the accuracy with which they report these activities and the physical operation of electrical devices. This paper carries out a comparison between self-reported energy-related activities and monitored electricity demand in 15 households over a week-long time period, with focus on electric hot water cylinders and heat pumps as appliances with large potential for demand flexibility. This comparison quantifies the extent to which selfreported activity is a predictor of electricity demand and conversely, whether electricity demand can accurately identify occupant activity. Results show that,
\end{abstract}

\footnotetext{
${ }^{1}$ Present address: Energy Centre, University of Auckland, 12 Grafton Rd, Auckland 1010, New Zealand
}

Preprint submitted to Energy and Buildings

October 5, 2018 
although there is significant variation across households, self-reported activity tends to be a reasonably good predictor of electricity demand. However, due to the intervention of thermostat-controlled devices, electricity demand is not a good indicator of occupant activity.

Keywords: time-use diary, residential electricity demand, demand flexibility

1 1. Introduction

2 The collective effect of many households using energy-intensive electrical 3 appliances at the same time can contribute to peak demand on the electricity 4 network $[1,2,3]$. Increasing uptake of new devices such as electric vehicles poses 5 risks of further increasing peak loads [4]. In addition, the rapid growth in non- dispatchable renewables, such as solar photovoltaics and wind [5] are likely to 7 exacerbate the temporal mismatch between supply and demand, causing many 8 countries to consider how future power systems might be managed [6, 7]. Tradi9 tionally, supply-side measures and demand management in the industrial sector have been used for balancing supply and demand, but there is an increasing interest in the potential role of demand flexibility - the modification of the time at which electricity demand occurs - in other sectors [8, 9, 10, 11, 12, 13].

The rollout of smart meters alongside the increased use of sensing and communications technology in household appliances (e.g. smart thermostats) has led to recent interest in the opportunity for demand flexibility in the residential sector $[12,14]$. Given the ingrained cultural relationship between people and their devices [15], an understanding of households' energy-related activities and how they interact with energy consuming appliances is required to unlock the full potential of residential demand flexibility [16, 17].

Time-use diaries (TUD) are a common method of trying to understand the activities of occupants in households [18, 19, 20, 21, 22, 23, 24]. These diaries typically involve occupants reporting their main and secondary activities in the household [20], and they are becoming popular tools for identifying correlations between reported activities and electricity demand at an aggregated regional 
or national level $[18,25,24]$. Data collected through TUDs is also being used to develop simulation models for predicting residential electricity demand at aggregated levels; by simulating a change in behaviour these models help explore opportunities for residential demand flexibility [20, 21, 26, 27, 28, 29, 30].

Reliance on TUD data for understanding energy-related household activity and simulating demand and demand flexibility rests on the assumptions that: (a) people accurately report their energy-related activities, and (b) their reported activities are directly associated with specific energy consumption by particular appliances [31]. However, with the exception of Widen et al.'s qualitative comparison of household level time use model-based and demand data [20, p760]; the quantitative comparison of aggregated customer data with modeled demand [20, p763] and recent small-scale studies in the health sciences [32, 33, 34] the validity of these assumptions remains largely untested and so the validity of the time-use derived models is uncertain [30].

An alternative approach which exploits the growing availability of smart meter data has lead to the development of methods to use total-house electricity consumption data to infer residents' activities [1]. These methods use statistical approaches to extract end-use and/or appliance level data from aggregate, or whole-building, electricity demand data. It has been proposed, for example, that the information extracted by these methods could be used to improve the representation of consumer behaviour in energy models [35]. However, there is an important distinction between identification of appliances - which is the focus of these methods - and identification of householder activities. Currently the relationship between people's everyday activities and the energy consumption of appliances is not well understood, particularly as many electrical appliances operate either autonomously or automatically [31]. The electricity demand of appliances that operate autonomously, e.g. via a thermostat, are by their nature somewhat decoupled from occupant activities, however it is often not clear to what extent this occurs. There is therefore a need for more precise quantification of how well occupant activity can or cannot be associated with measured electricity demand at the household level. 
This paper responds to these problems by reporting a novel analysis of combined household time-use survey data and high-granularity, circuit-level electricity demand data to answer the following key questions: "Are reported activities a good predictor of appliance level electricity demand and conversely "Is appliance-level electricity demand a good predictor of occupant activities?" In this paper we address these questions in the specific case of heat pumps and hot water cylinders in New Zealand. In doing so we explicitly respond to McKenna et al.'s call for studies that examine the link between time-use activities and actual energy demand [30] and especially the need to collect and understand "data describing the relationship between activities and appliance energy use, and how this varies within and between households." [30, p. 14]. To do this we use data from a study of 15 houses each of which used a time-use diary to collect household members' reports of their energy-related activities, and circuit-level monitored electricity demand (power) data at one minute resolution [36]. The paper reports on a detailed comparison of these data sets to quantify the extent to which self-reported activity can be a predictor of electricity demand and conversely, electricity demand a predictor of occupant activity. For the purposes of this paper we analysed only the data relating to electrical heating of hot water and the use of heat pumps for space heating. The broader study collected data on many other activities and also included the use of gas for water and space heating, but as our focus is on electricity use in households, we have omitted these in the present study.

This paper is organised as follows: section 2 explores the demand flexibility opportunities from heating loads, section 3 presents the data sets that were used for the current study, and section 4 describes the methodology we have applied to compare the TUD and electricity demand data sets. Section 5 presents the results of the comparison, which are discussed along with the conclusions in section 6. 


\section{Heating and demand flexibility}

85 Due to their relatively large electricity demand and energy storage potential,

s6 thermo-electric appliances are of increasing interest for shifting demand [8,

87 37, 38, 39]. These appliances offer significant potential for demand flexibility

s8 because they enable a large load to be shifted while minimizing the impact on

89 service provision [8]. In this work we focus primarily on electrical water heaters and heat pumps.

Electric hot water cylinders for domestic hot water have a high penetration in many countries [40, 41], and account for a large proportion of demand especially during peak times. For example, in New Zealand hot water cylinders are present in $88 \%$ of households, where they make up $30 \%$ of daily electricity demand and $50 \%$ of morning and evening peak demand [42]. Typical hot water cylinders have the capacity to store roughly $10 \mathrm{kWh}$ of heat energy and are usually operated fully autonomously via a thermostat with pre-set temperature settings. In a flexible demand scenario, a smart controller can be used to override the thermostat and shift electricity demand to other times with potentially no impact on the hot water consuming activities of the occupants [42].

Analysis of the time lag between drawing hot water from the tank and the heating element engaging to restore the temperature in New Zealand hot water cylinders has shown it to be in the range of a few minutes [42]. Hence a longer draw such as a warm shower will cause the element to switch on soon after the activity begins, whereas smaller loads, such as washing of hands, may not trigger the hot water cylinder at all, which may artificially lead to such activities having a comparatively low probability of hot water cylinder power draw, given the activity is reported.

The use of heat pumps for space heating and cooling has experienced significant growth over the last few decades in many countries. Heat pumps have a more complicated operation than hot water cylinders in that, in addition to a thermostat, they can also be switched off and on by the user, which can lead to significant variability in use of heat pumps in households. Unlike many 
countries, in New Zealand, heat pumps are to a large extent controlled manually by the occupants. In particular, they are often turned off during the day, when the occupants leave the house, and at night when the occupants go to bed [43]. This mode of operation makes the relationship between the activities of the occupants, e.g. turning on the heat pump, and electricity demand quite complex.

Heat pumps make a substantial contribution to peak demand in many countries and there have been a number of proposals to control the use of heat pumps during these peak periods [44] through reducing (when heating) or increasing (when cooling) the thermostat setting for short periods of time and therefore reducing power demand. These proposals assume that houses will have sufficient thermal mass that this reduction in thermostat temperature will have only a minor impact on indoor temperature. In New Zealand it is not necessarily the case due to lack of effective insulation in many houses.

While both hot water cylinders and heat pumps operate in on/off mode, with heat pumps the power draw depends on the indoor and outdoor temperature difference and the temperature setting-lowering the temperature setting will result in a decreased electricity draw. Therefore heat pumps can potentially be used for peak shaving, if incentives are put in place to encourage householders to change their times of heating or alter thermostat levels on request. Hot water cylinders can be used for demand response all year round, whereas in New Zealand heat pumps are used mainly in winter for on-demand space heating and are generally manually controlled so that greatest demand tends to be during morning and evening peak periods. Hence both appliances have potential to be used for load shifting or peak shaving. Understanding if and how this potential could be realized, whether through smart control while maintaining services within user preferences, or through behaviour change, requires a better understanding of the relationship between household activities at different times of day and the electricity consumption of these appliances. 


\section{Time-use diary and monitored electricity demand data sets}

Circuit-level electricity demand data with one minute time resolution was collected for 15 households. This is a subset of a larger data set collected within the GREEN Grid project $[45,36]$ in the Hawke's Bay region in New Zealand's North Island. The 15 households comprise of the subset that had (1) electricity demand data, (2) TUD data and (3) an electric water heating and/or a heat pump. Of the 15 houses, 12 households had electric water heating and 10 households had heat pumps. Table 1 gives a summary of the collected data sets from these households.

Hot water cylinders and heat pumps were monitored on their own circuits, where possible. During the monitoring period, all occupants of the participating households were also requested to report all their energy-related activities in a time-use diary over a week starting at 06:00 on a Monday and finishing at 06:00 seven days later. A total of 59 people (32 adults and 27 children under the age of 18) lived in the 15 houses. Of these, 34 people reported activities.

The time intervals in the time-use diary (TUD) study were: 15 minutes between 07:00 and 09:00, and 17:00 and 20:00 (periods of peak consumption nationally), a night time interval of six hours between midnight and 06:00, and 30 minute time intervals at all other times.

For the time-use diaries, participants were asked to record all of their activities (examples were provided) that involved the use of energy (e.g. electricity, gas, wood, coal, solar) as well as activities that avoided the use of extra energy (such as drawing curtains to keep in warmth, or putting on additional clothing rather than turning up the heat pump). They were able to record several activities occurring simultaneously. A separate section on the use of washing machines asked them to record the start time of the wash, size of the wash and water temperature.

Table 2 gives the reported activities relating to the use of hot water, and the number of times those activities were reported in the TUD data set. For heat pumps, participants were requested to report if they turned their heat pump(s) 


\begin{tabular}{|c|c|c|c|c|c|}
\hline Data set & Description & $\begin{array}{l}\text { Time } \\
\text { period } \\
\text { covered }\end{array}$ & $\begin{array}{l}\text { Number } \\
\text { of house- } \\
\text { holds }\end{array}$ & $\begin{array}{l}\text { Original } \\
\text { data } \\
\text { resolution }\end{array}$ & $\begin{array}{l}\text { Analysed } \\
\text { data } \\
\text { resolution }\end{array}$ \\
\hline $\begin{array}{l}\text { Electricity } \\
\text { monitor- } \\
\text { ing }\end{array}$ & $\begin{array}{l}\text { Monitored } \\
\text { load }(\mathrm{W}) \\
\text { at } \\
\text { household } \\
\text { circuit } \\
\text { level }\end{array}$ & $\begin{array}{l}\text { May } 2014 \\
\text { to } \\
\text { present; } \\
\text { varies by } \\
\text { household }\end{array}$ & $\begin{array}{l}15, \text { of } \\
\text { which } 12 \\
\text { with } \\
\text { electric } \\
\text { hot water, } \\
\text { and } 10 \\
\text { with heat } \\
\text { pumps }\end{array}$ & 1 minute & $\begin{array}{l}30 \\
\text { minutes } \\
(\text { mean W) }\end{array}$ \\
\hline $\begin{array}{l}\text { Time-use } \\
\text { diaries } \\
\text { (TUD) }\end{array}$ & $\begin{array}{l}\text { Self- } \\
\text { reported } \\
\text { energy- } \\
\text { related } \\
\text { activities } \\
\text { by each } \\
\text { occupant } \\
\text { of } \\
\text { household }\end{array}$ & $\begin{array}{l}20.07 .2015 \\
26.07 .2015 \\
(1 \text { week })\end{array}$ & $\begin{array}{l}15 \text { (as } \\
\text { above) }\end{array}$ & $\begin{array}{l}\text { Intervals } \\
\text { of } 15 \\
(07: 00- \\
09: 00 \text { and } \\
\text { 16:00 - } \\
20: 00) \text { or } \\
30 \\
\text { minutes } \\
\text { (all other } \\
\text { periods) } \\
\text { from } \\
\text { 06:00 to } \\
00: 00\end{array}$ & $\begin{array}{l}30 \\
\text { minutes } \\
(15 \text { min } \\
\text { intervals } \\
\text { merged to } \\
30 \text { min } \\
\text { intervals) }\end{array}$ \\
\hline
\end{tabular}

Table 1: Data sets and their main characteristics.

173 on or off, and give a "thermostat setting, if changed".

$174 \quad$ Figures 1 and 2 provide examples of the two superimposed data sets for two 175 of the houses, showing the measured electricity use (lines) and reported activ- 


\begin{tabular}{|l|l|}
\hline Activity & Number of instances \\
\hline \hline Shower & 167 \\
Dishes, by hand & 66 \\
Bath & 31 \\
Wash using basin & 23 \\
Laundry using hot water & 7 \\
Clean personal items & 2 \\
\hline
\end{tabular}

Table 2: Activities related to hot water, and corresponding total number of instances in the data set.

ities (dots). With hot water use (Figure 1) our main focus was on reported showers and baths, because they cause a clear electricity draw from the hot water cylinder that can be relatively easily distinguished from maintenance draws, where the thermostat inside the hot water cylinder induces a power draw automatically when the water temperature drops below a certain value. However, we have also recorded and analysed smaller-scale hot water usage events such as washing dishes by hand. With heat pumps (Figure 2) we included reports of turning on or off the appliance and adjusting the temperature.

A number of features can be observed directly from these plots. For example in Figure 2, a clear electricity draw coincides with a TUD record of turning on a heat pump, and often also an immediate decrease of power draw to a zero coincides with a TUD record of turning off a heat pump. However, there are also several instances where an activity has been reported, but no corresponding power draw is visible, and vice versa. In the next section we explore a method of quantifying these observations. 

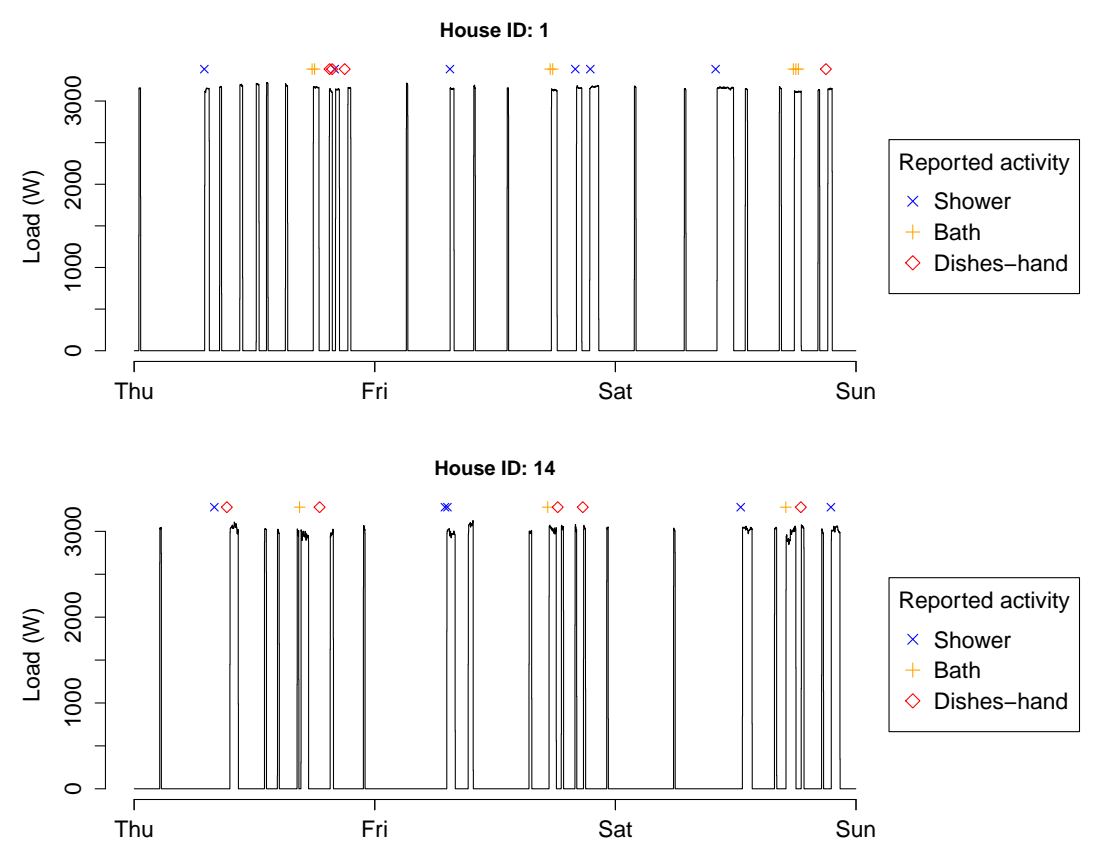

Figure 1: Example of data on hot water cylinder power draws and reported activities during three days for two houses. 


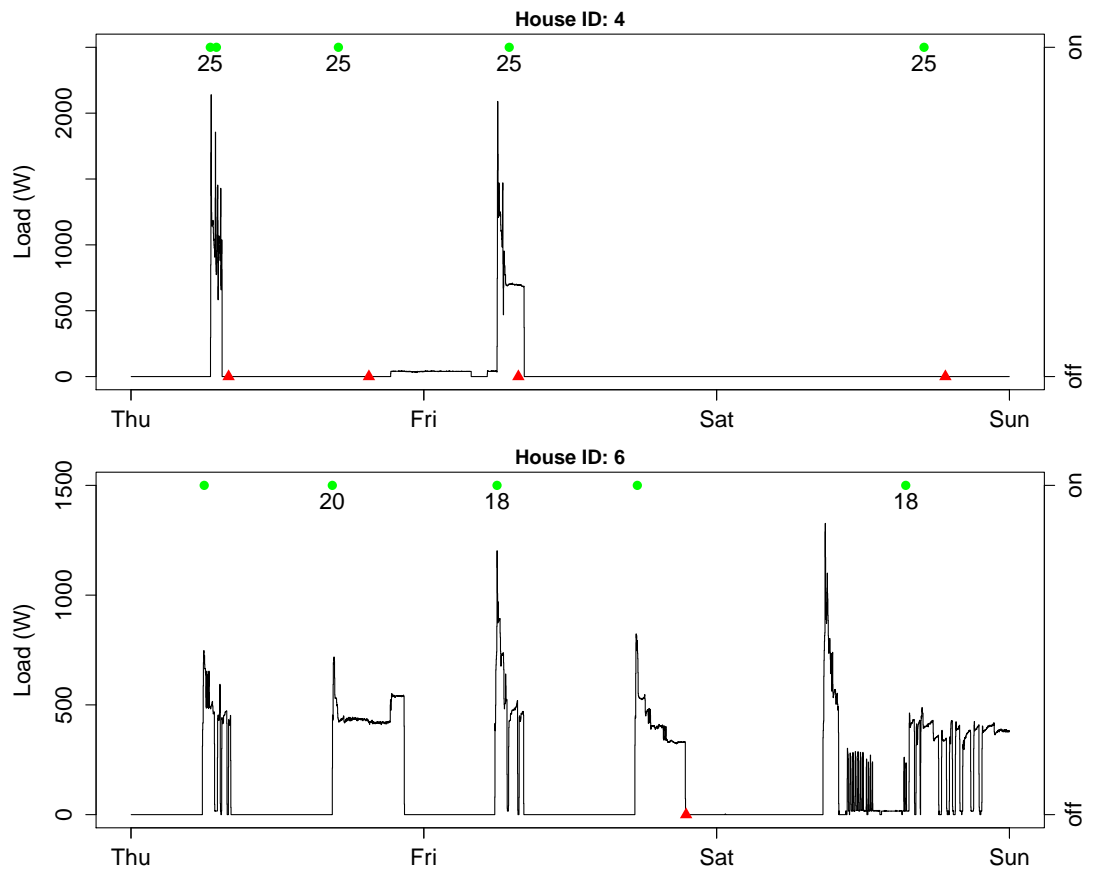

Figure 2: Example of data on heat pump usage and reported activities over three days for two houses. Green dots (upper right-hand scale) indicate a reporting of turning on the heat pump, or adjusting the setting (temperature setting given below, if reported), and the red triangles (lower right-hand scale) indicate a reporting of turning off the heat pump. 


\section{Methodology for comparison of reported time use activities and monitored electricity demand}

In this section we describe the methodology used to make a detailed comparison of the TUD and monitored electricity time-series data sets.

To understand the relationship between reported activities and demand we created two time-series of uniform 30-minute time-periods (e.g. 07:00-07:30) from the underlying power and time use data. In the case of the electricity demand data, we derived the mean power demand for each appliance (hot water or heat pump) in each half-hour. For the time-use diary data we determined if an activity had been reported in the relevant half-hour slot. As table 1 shows this meant aggregating time-use activities recorded in the 15-minute periods (07:00-09:00 and 16:00-20:00) to create uniform 30-minute records for the day (06:00-00:00) which recorded whether or not a relevant activity had been recorded. Although the 15-minute level time-use data would have enabled a higher granularity test of the coincidence of time-use reporting and actual demand, excluding the half-hourly time-use data would have reduced the number of such recordings substantially making the analysis infeasible. Since no activities were reported during the 00:00-06:00 period, no time-use data was excluded.

Using this derived data set we can determine the number of instances of time slots where an activity was reported coincident with a certain level of electricity demand. If we denote no activity reported in a particular time slot as $A_{0}$, one or more activities as $A_{1}$ and electricity demand during the time slot in the $k$ th level of $\mathrm{kWh}$ values as $d_{k}$, then the number of instances of having both an activity reported and measuring an electricity demand in the $k$ th level is given by $F\left(A_{1} \wedge d_{k}\right)$. If the reported activity is an indicator of electricity demand then the number of instances of time slots with reported activities will be higher for higher levels of electricity demand. Similarly we can also determine the number of instances of time slots with no activity reported. In this case the number of instances of having both no activity reported and having a electricity demand in 
the $k$ th level is $F\left(A_{0} \wedge d_{k}\right)$. We expect this to show that the number of instances of time slots with no activity will be higher for lower levels of electricity demand.

For our current purposes electricity demand is considered non-zero if the demand averaged over the time slot is greater than a certain threshold $d_{\mathrm{th}}$. Here we take this threshold to be at $10 \%$ of the maximum value of the averaged demands for each time slot over the full time period. This is an arbitrary cut-off but has been verified to be a reasonable choice by studying the load distribution plots of the households in further detail, which show a distinct gap between the number of instances of loads within the lowest $10 \%$ and higher loads.

\subsection{Conditional probabilities}

In contrast to Widen et al.'s aggregated household demand approach [20, p763], research questions "Are reported activities a good predictor of appliancelevel electricity demand?" and "Is appliance-level electricity demand a good predictor of occupant activities?" at the household level are most clearly formulated in terms of conditional probabilities. For example, to answer the first question we are interested in establishing the probability that there is a non-zero demand in a certain time slot, given that an activity is reported in that time slot. Or similarly, the probability that there is a non-zero demand in a certain time slot, given that no activity is reported in that time slot. To answer the second question, we would like to know if observed electricity demand can be used as a predictor for an activity, or if the absence of electricity demand means that no activity is taking place.

Denoting zero electricity demand as $D_{0}: d_{k}<d_{\text {th }}$ and a non-zero demand as $D_{1}: d_{k} \geq d_{\mathrm{th}}$, we can then formulate the following conditional probabilities as given in table 3 .

Conservation of probability requires the following relationships between the conditional probabilities: $P\left(D_{0} \mid A_{1}\right)=1-P\left(D_{1} \mid A_{1}\right), P\left(D_{0} \mid A_{0}\right)=1-P\left(D_{1} \mid A_{0}\right)$, $P\left(A_{1} \mid D_{0}\right)=1-P\left(A_{0} \mid D_{0}\right)$ and $P\left(A_{1} \mid D_{1}\right)=1-P\left(A_{0} \mid D_{1}\right)$. 


\begin{tabular}{|c|c|c|}
\hline $\begin{array}{c}\text { Conditional } \\
\text { probability }\end{array}$ & Probability of & Condition \\
\hline$P\left(D_{1} \mid A_{1}\right)$ & non-zero electricity demand & an activity is reported \\
$P\left(D_{0} \mid A_{1}\right)$ & zero electricity demand & an activity is reported \\
$P\left(D_{1} \mid A_{0}\right)$ & non-zero electricity demand & no activity is reported \\
$P\left(D_{0} \mid A_{0}\right)$ & non-zero electricity demand & no activity is reported \\
$P\left(A_{1} \mid D_{1}\right)$ & an activity is reported & non-zero electricity demand \\
$P\left(A_{0} \mid D_{1}\right)$ & no activity is reported & non-zero electricity demand \\
$P\left(A_{1} \mid D_{0}\right)$ & an activity is reported & zero electricity demand \\
$P\left(A_{0} \mid D_{0}\right)$ & no activity is reported & zero electricity demand \\
\hline
\end{tabular}

Table 3: Conditional probability denotations and their respective conditions.

For two events $A$ and $B$ the conditional probability is given by

$$
P(A \mid B)=\frac{P(A \wedge B)}{P(B)}
$$

250

251

252

253

254

where $P(A \wedge B)$ is the probability of both event $A$ and $B$ occurring and $P(B)$ is the total probability of event $B$ occurring. Applied to our data sets, the probabilities $P\left(A_{j} \wedge D_{i}\right)$, and $P\left(A_{j}\right)$ and $P\left(D_{j}\right)$ where $j=0,1$ and $i=0,1$ can be approximated from the number of instances $(F)$ of activity or no activity versus electricity demand level, as described in the previous section, i.e.

$$
\begin{aligned}
P\left(A_{j} \wedge D_{i}\right) & \approx \frac{F\left(A_{j} \wedge D_{i}\right)}{N} \\
P\left(A_{j}\right) & \approx \frac{F\left(A_{j}\right)}{N} \\
P\left(D_{i}\right) & \approx \frac{F\left(D_{i}\right)}{N}
\end{aligned}
$$

where $N$ is the total number of time slots over the whole one-week time period. 


\section{Results}

This section shows the results of applying the above methodology to the two data sets.

\subsection{Hot water cylinders}

Table 4 gives the number of instances (during the week-long period) of time slots when a hot water related activity was either reported or not and a particular electricity demand was measured. We have presented the results considering both 30 or 60 minute time slots and the electricity demand has been normalised to the maximum of average load in all time slots over the week i.e. first the average load for each 30 or 60 minute period is calculated, and those values are then normalised to the maximum value of those average loads. For example, using a 30 minute time slot, 93 time slots had activities reported where the average load was below $10 \%$ of the maximum load (and hence considered to have zero load for our purposes).

Figure 3 presents the data in table 4 in graphical form. This shows that when the time series is considered in 30 minute time slots, most activities are either reported when a significant load is observed (last bin) or when very little or no load is observed (first bin). In the bins in between, the hot water cylinder is either ramping up or turning off during that time slot, meaning it is on for only a fraction of the time slot. When the time series is considered in 60 minute time slots, this distinction disappears, as it is unusual for the hot water cylinder to be at maximum load for the full hour. Activities reported in the first bin essentially indicate a misreported activity — either the timing is wrong, or no activity occurred. Activities reported in all other bins imply a load occurred in that time interval, and hence the activity was accurately reported.

Table 5 gives the conditional probability results for the hot water cylinders in the 12 houses with electric hot water cylinders. It shows the results for all hot water related activities, i.e. based on the results in table 4 . Table 6 shows the results for each activity individually. The number in parenthesis in column one 

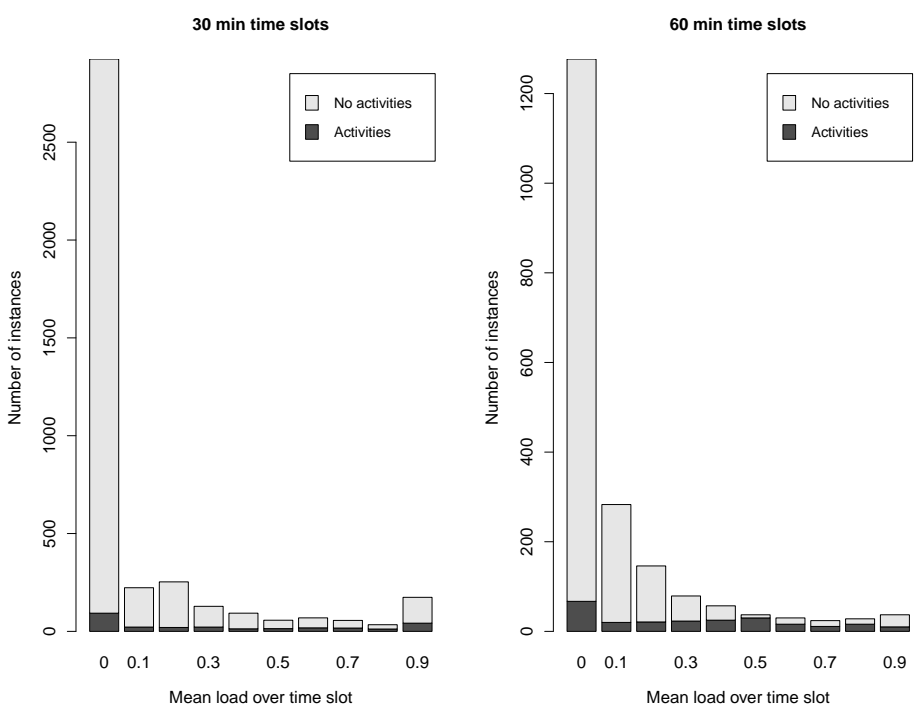

Figure 3: Number of instances of 30-minute (left) and 60-minute (right) time slots with (dark grey) and without (light grey) hot water reported activities at each value of measured electricity demand (normalised) for all 12 houses with electric water heating. The actual numbers can be found in Table 4 . 


\begin{tabular}{|c|r|r|r|r|r|r|r|r|r|r|}
\hline Load (normalised) & 0 & 0.1 & 0.2 & 0.3 & 0.4 & 0.5 & 0.6 & 0.7 & 0.8 & 0.9 \\
\hline 30 min, activity & 93 & 22 & 20 & 22 & 13 & 14 & 18 & 17 & 12 & 42 \\
30 min, no activity & 2832 & 201 & 233 & 106 & 80 & 43 & 51 & 39 & 22 & 132 \\
60 min, activity & 67 & 20 & 21 & 23 & 25 & 30 & 16 & 11 & 16 & 10 \\
60 min, no activity & 1210 & 263 & 125 & 56 & 32 & 7 & 14 & 13 & 12 & 27 \\
\hline
\end{tabular}

Table 4: Number of instances for hot water use for the 12 houses: the number of time intervals with activities (top two rows) and no activities (bottom two rows) reported per normalised average load in that time slot.

gives the number of reported activities in each group. Column three, $P\left(D_{1} \mid A_{1}\right)$, shows that showers, the most commonly reported activity, are also the most accurately reported activity, reported correctly $73 \%$ of the time with 30 -minute time intervals, and $79 \%$ of the time with 60 -minute time intervals. Torriti [19] found that out of six social practices (or household activities) washing has the highest time dependence, especially during week days. It is possible that participants found it easier to accurately report the timing of showers because of their routine nature.

Increasing the time slot for each reported activity to 60 minutes increases the probability of observing a load in that time slot, as can be expected due to cases of remembering to report the activity, but getting the timing somewhat wrong. Doing dishes by hand and bathing have a relatively high reporting accuracy; at 30 minute intervals reporting accuracy is correct just over $64 \%$ and $56 \%$ of the time, respectively, increasing to $71 \%$ and $63 \%$ at one hour time resolution. The results indicate that reporting of these activities can be a reasonable predictor of load.

The results for washing using the basin, e.g. washing hands or face, are between $35 \%$ and $40 \%$ for all considered time intervals, indicating either very inaccurate reporting, or, that the duration was too short to initiate a significant electricity load in the hot water cylinder. There were only seven reports of 
laundry, after cold washes were omitted. The accuracy of reporting correctly increases from one third of the time, to two thirds of the time in going from $30 \mathrm{~min}$ to $60 \mathrm{~min}$ time slots, which could be explained by the time use diary inquiring about laundry separately on the last page of the diary for each day, and hence often being filled only at the end of each day rather than when the activity occurred. Hence, for various reasons, there is too much uncertainty around the reporting of these activities and their correlation with measured load to serve as a predictor of electricity demand.

The probability of a non-zero load in time intervals where no activity is reported, $\left(P\left(D_{1} \mid A_{0}\right)\right)$, reflects the typical functioning of a hot water cylinder; it can often stay on for a longer period than the determined time slots. Also, regular maintenance events will increase this probability. In other words, there will always be a certain probability of the hot water cylinder drawing electricity, even when no activity is occurring.

The last two columns give the results for the second set of conditional probabilities; looking at whether an observed load is a good predictor of activities. The results for $P\left(A_{1} \mid D_{1}\right)$ show that the overall probability of an activity being reported, given a load is observed, is less than $17 \%$ at 30 minute intervals, and below $24 \%$, when looking at 60 minute time intervals. Thus, observed load is not a good predictor of activities. However, the absence of load is a good predictor of the absence of activities, as shown in the last column.

Calculating $P\left(D_{1} \mid A_{0}\right), P\left(A_{1} \mid D_{1}\right)$ or $P\left(A_{1} \mid D_{0}\right)$ for individual activities is not possible, because it is not possible to differentiate between different activities based on the observed loads.

To look at differences in reporting accuracy between households, the conditional probabilities were also calculated for each household. Figure 4 shows $P\left(D_{1} \mid A_{1}\right)$ for each house for both 30 and 60 minute time resolutions considering all activities, ordered from highest to lowest at the 30 minute time resolution. The results show that reporting accuracy varies from very poor at below $20 \%$ to very good at $100 \%$ at 30 minute time resolution, and increases slightly for most households when increasing the observed time slot. Most households show 


\begin{tabular}{|c|c|c|c|c|c|}
\hline Activity & Time interval & $P\left(D_{1} \mid A_{1}\right)$ & $P\left(D_{1} \mid A_{0}\right)$ & $P\left(A_{1} \mid D_{1}\right)$ & $P\left(A_{1} \mid D_{0}\right)$ \\
\hline \hline \multirow{2}{*}{ All } & $30 \mathrm{~min}$ & 0.659 & 0.243 & 0.166 & 0.032 \\
& $60 \mathrm{~min}$ & 0.720 & 0.312 & 0.239 & 0.052 \\
\hline
\end{tabular}

Table 5: Conditional probabilities for all activities related to hot water cylinders.

\begin{tabular}{|c|c|c|}
\hline Activity & Time interval & $P\left(D_{1} \mid A_{1}\right)$ \\
\hline \hline Showers & $30 \mathrm{~min}$ & 0.728 \\
$(167)$ & $60 \mathrm{~min}$ & 0.791 \\
\hline Dishes, hand & $30 \mathrm{~min}$ & 0.635 \\
$(66)$ & $60 \mathrm{~min}$ & 0.710 \\
\hline Baths & $30 \mathrm{~min}$ & 0.560 \\
$(31)$ & $60 \mathrm{~min}$ & 0.625 \\
\hline Wash, basin & $30 \mathrm{~min}$ & 0.391 \\
$(23)$ & $60 \mathrm{~min}$ & 0.364 \\
\hline Laundry & $30 \mathrm{~min}$ & 0.333 \\
$(7)$ & $60 \mathrm{~min}$ & 0.667 \\
\hline
\end{tabular}

Table 6: Conditional probabilities of individual activities related to hot water cylinders. The number of recorded instances of each activity is given in parenthesis.

a reporting accuracy of $60-80 \%$ at the 30 minute time resolution.

\subsection{Heat pumps}

The use of heat pumps by occupants was reported as "turning the heat pump on", sometimes reported with a temperature setting, or "turning the heat pump off". The accuracy of reporting is measured as observations of non-zero

load in the time interval where the heat pump was reported to be turned on. Table 7 quantifies the number of occurrences of reporting "turning the heat 


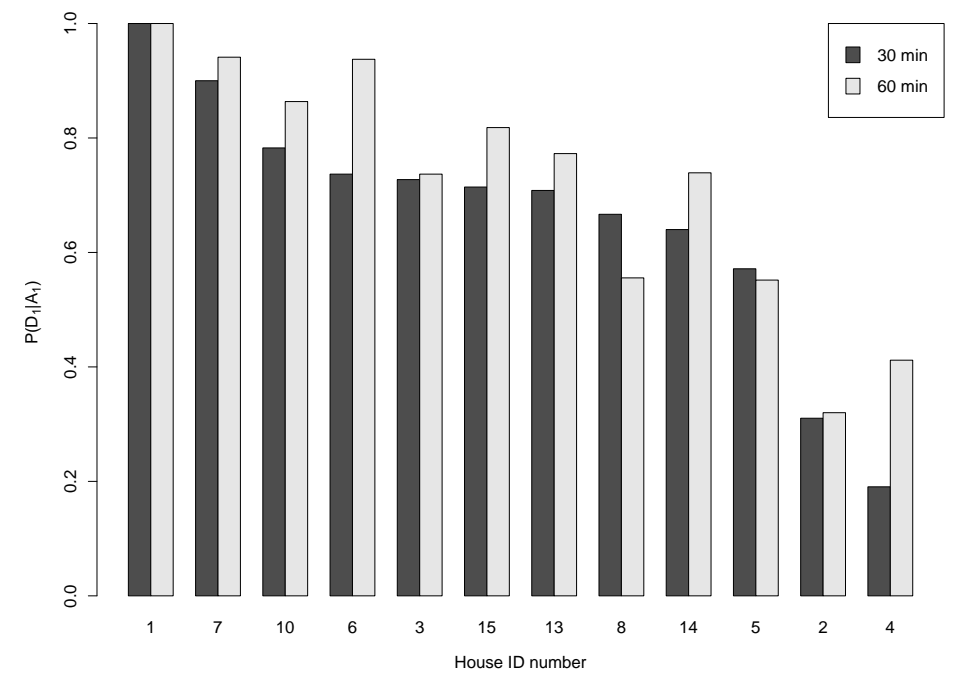

Figure 4: Conditional probability $P\left(D_{1} \mid A_{1}\right)$ for hot water cylinders for each house.

\begin{tabular}{|r|r|r|r|r|r|r|r|r|r|r|}
\hline & 0 & 0.1 & 0.2 & 0.3 & 0.4 & 0.5 & 0.6 & 0.7 & 0.8 & 0.9 \\
\hline 30 min, activity & 24 & 16 & 22 & 18 & 18 & 11 & 10 & 9 & 1 & 1 \\
30 min, no activity & 2198 & 250 & 302 & 183 & 71 & 100 & 68 & 32 & 16 & 2 \\
60 min, activity & 17 & 25 & 31 & 22 & 10 & 4 & 10 & 7 & 0 & 0 \\
60 min, no activity & 1067 & 124 & 145 & 94 & 29 & 46 & 29 & 10 & 4 & 0 \\
\hline
\end{tabular}

Table 7: Number of instances for heat pumps: the number of time slots with activities (top two rows) and no activities (bottom two rows) reported per normalised load in that time slot.

pump on", considering time slots of 30 and 60 minutes. Figure 5 is a graphical representation of table 7 .

Table 8 gives the conditional probability results for the use of heat pumps in the 10 houses with heat pumps, based on the results in table 5 . The results show that the probability of reporting the activity ("turning the heat pump on") correctly, i.e. coinciding with non-zero load from the heat pump, is quite high, with approximately $82 \%$ accuracy at 30 minute time resolution, and approxi- 

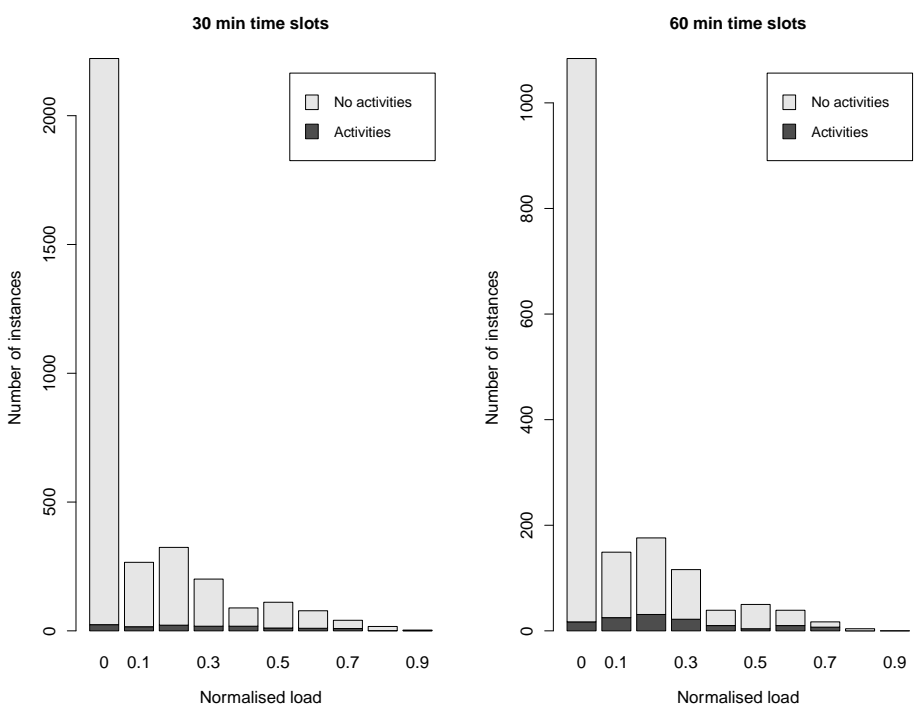

Figure 5: Number of instances of 30-minute (left) and 60-minute (right) time slots with (dark grey) and without (light grey) heat pump reported activities at each value of measured electricity demand (normalised). The actual numbers can be found in table 7 .

mately $87 \%$ accuracy when increasing the time intervals to 60 minutes. This indicates that the reported activities are a reasonable predictor of demand.

The over $30 \%$ probability for a non-zero load even when no activity is reported $\left(P\left(D_{1} \mid A_{0}\right)\right)$, is explained by the way a heat pump operates; staying on until turned off, or turning itself off only when the set temperature is reached. However, it is not possible to distinguish whether a non-zero load is due to the heat pump being on from a previous activity, or whether an activity has been misreported, i.e. forgotten to be reported, or simply getting the timing wrong. The low probabilities for $P\left(A_{1} \mid D_{1}\right)$ indicate that demand is not a good predictor of activities.

Figure 6 gives the conditional probabilities per household. Three of the households show perfect reporting of their heat pump usage at both 30 and 60 minute time resolutions. House 12 has the overall lowest reporting accuracy. Houses 6 and 11 show higher reporting accuracy at 30 minute time resolutions, 


\begin{tabular}{|c|c|c|c|c|}
\hline Time interval & $P\left(D_{1} \mid A_{1}\right)$ & $P\left(D_{1} \mid A_{0}\right)$ & $P\left(A_{1} \mid D_{1}\right)$ & $P\left(A_{1} \mid D_{0}\right)$ \\
\hline \hline $30 \mathrm{~min}$ & 0.815 & 0.318 & 0.094 & 0.011 \\
$60 \mathrm{~min}$ & 0.865 & 0.311 & 0.185 & 0.016 \\
\hline
\end{tabular}

Table 8: Conditional probabilities for heat pumps.

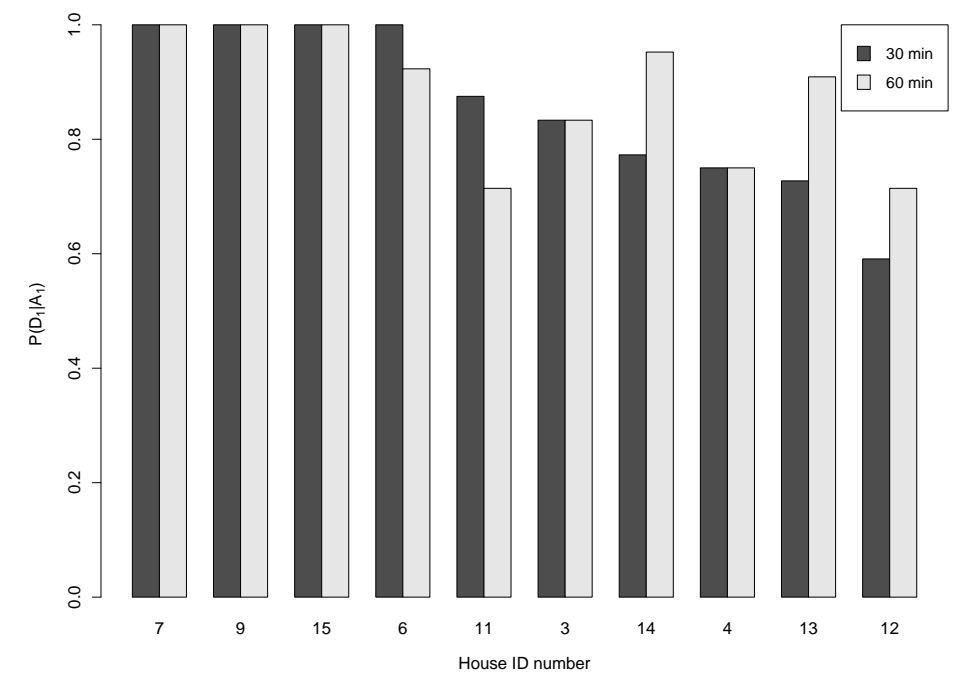

Figure 6: Conditional probabilities $P\left(D_{1} \mid A_{1}\right)$ for heat pumps for each house.

which can be explained by observing the load time series. These show the heat pumps being used for very short durations at a time, which average below $10 \%$ of maximum average load. Hence, it is not an indication of inaccurate reporting. Overall, the reporting accuracy in generally well above $70 \%$ at both 30 and 60 minute time resolutions.

\section{Discussion and conclusions}

The aim of this paper was to respond to some of the shortcomings in current activity based energy demand models as identified by McKenna et al. [30] and quantify to what extent reported activities related to hot water and heat 
pump usage can predict electricity demand of the corresponding appliances and conversely to what extent electricity demand of those appliances is a good predictor of household occupant activities. The collected data sets enable us to determine how accurately people report their energy-related activities, and how well their reported activities relate to measured consumption by appliances. These are important to understand if time-use diaries are to be reliably used for modelling residential opportunities for demand flexibility and if measured electricity demand can be reliably used for inferring household occupants' activities, respectively [30].

To do this we developed a novel methodology for systematic comparison between time-use diaries and electricity demand data which enables the quantification of household-level correspondence. This goes beyond previously reported qualitative household level [20, p760] and quantified but indirect aggregated household demand validation approaches [20, p763].

The results show that at a 30 minute resolution participants were accurate approximately $66 \%$ of the time when reporting hot-water-related activities and approximately $82 \%$ of the time when reporting heat pump usage. Occasionally, reporting an activity, particularly turning off the heat pump as visually observed in the electricity demand data, was not reported, which suggests something about the psychology of energy use; switching on a device appears to be given greater emphasis - as evidenced by the fact that it is more likely to be written down - than switching off an appliance, at least in the case of heat pumps. However, it is also possible the heat pump switched itself off after reaching a set temperature, something we can not distinguish with the current data. Other human factors that may affect the accuracy of reporting relate to the way the study was conducted. For example, some participants reported filling in the diary throughout the day, whereas others filled it in at the end of the day or later, and sometimes they relied on another family member to fill it in for them. In summary, our results show that reported activities related to the use of hot water and heat pumps are a reasonable predictor of non-zero demand in New Zealand, but the absence of a reported activity is not a good predictor of 
zero demand. The reliability of the data and thus of any subsequent demand models is dependent on the close alignment of occupant activity and subsequent power demand which may be unusually highly correlated in New Zealand due to the combination of specific appliances and the way they are used. This is especially true for heat pump use which shows the highest reliability (82\%) and suggests that similar studies carried out in response to McKenna et al.'s call [30] but in other socio-technical contexts may find far lower levels of correspondence.

Reliability is also driven by the accuracy of reporting, and our work recommends the use of simpler and less time-consuming approaches than hand written diaries to reporting activities, such as applications on a smart phone [22], or some other conveniently used device for quick and explicit reporting. In addition, due to the limited sample size of our study the results should not be taken as representative of reporting accuracy at a national level. Rather, they motivate undertaking a larger study for national level verification and applicability across broader geographical areas.

While reported activities were relatively good predictors of demand, this study found that monitored electricity demand of hot water cylinders and heat pumps is not a good predictor of recorded householder activities. The probability of an activity being recorded given that an electricity demand is observed during a 30 minute interval was less than $20 \%$ in most cases. We can understand this finding from the fact that in the case of thermostat driven devices, determining the accuracy of 'no reported activity' is much more difficult as it is not possible to distinguish between human misreporting and automatic control due to presence of thermostats. Similar findings have been described by Durand-Daubin [31] and we conclude that extreme care must be taken when inferring household activities - as opposed to appliance use-from smart meter data at this level of granularity.

Overall, the results from our limited sample suggest that approaches based on national time-use diaries may be a valid approach to modeling residential demand and demand flexibility for the activities and appliances we have tested here. Further work is required to see if the results hold for representative na- 
436

437 Acknowledgements

$438 \quad$ Financial support for this research from the New Zealand Ministry of Busi-

439 ness, Innovation and Employment (Contract No. UOCX1203) is gratefully ac440 
441

442

443

444

445

446

447

448

\section{References}

[1] A. Kavousian, R. Rajagopal, M. Fischer, Determinants of residential electricity consumption: Using smart meter data to examine the effect of climate, building characteristics, appliance stock, and occupants' behavior, Energy 55 (2013) 184-194.

[2] G. Walker, The dynamics of energy demand: Change, rhythm and synchronicity, Energy Research \& Social Science 1 (2014) 49-55.

[3] J. Torriti, Peak Energy Demand and Demand Side Response, Routledge, London, 2015.

[4] T. Boßmann, I. Staffell, The shape of future electricity demand: Exploring load curves in 2050s Germany and Britain, Energy 90 (2015) 1317-1333.

[5] IRENA, Renewable capacity statistics 2017, Tech. rep., International Renewable Energy Agency (IRENA), Abu Dhabi (2017).

[6] G. Strbac, Demand side management: Benefits and challenges, Energy Policy 36 (12) (2008) 4419-4426.

[7] G. Strbac, I. Konstantelos, M. Aunedi, M. Pollitt, M. Green, Delivering future-proof energy infrastructure, Report for the National Infrastructure Commission, University of Cambridge \& Imperial College London (2016).

[8] P. D. Lund, J. Lindgren, J. Mikkola, J. Salpakari, Review of energy system flexibility measures to enable high levels of variable renewable electricity, Renewable and Sustainable Energy Reviews 45 (2015) 785-807.

[9] S. J. Darby, Smart electric storage heating and potential for residential demand response, Energy Efficiency (2017) 1-11.

[10] C. Eid, P. Codani, Y. Perez, J. Reneses, R. Hakvoort, Managing electric flexibility from Distributed Energy Resources: A review of incentives for market design, Renewable and Sustainable Energy Reviews 64 (2016) 237247. 
[11] N. Prüggler, Economic potential of demand response at household levelare Central-European market conditions sufficient?, Energy Policy 60 (2013) 487-498.

[12] R. D'hulst, W. Labeeuw, B. Beusen, S. Claessens, G. Deconinck, K. Vanthournout, Demand response flexibility and flexibility potential of residential smart appliances: Experiences from large pilot test in Belgium, Applied Energy 155 (2015) 79-90.

[13] P. Grünewald, M. Diakonova, Flexibility, dynamism and diversity in energy supply and demand: A critical review, Energy Research \& Social Science 38 (2018) 58-66.

[14] S. Nistor, J. Wu, M. Sooriyabandara, J. Ekanayake, Capability of smart appliances to provide reserve services, Applied Energy 138 (2015) 590-597.

[15] J. Stephenson, B. Barton, G. Carrington, A. Doering, R. Ford, D. Hopkins, R. Lawson, A. McCarthy, D. Rees, M. Scott, P. Thorsnes, S. Walton, J. Williams, B. Wooliscroft, The energy cultures framework: Exploring the role of norms, practices and material culture in shaping energy behaviour in New Zealand, Energy Research \& Social Science 7 (2015) 117-123.

[16] R. Ford, M. Pritoni, A. Sanguinetti, B. Karlin, Categories and functionality of smart home technology for energy management, Building and Environment 123 (2017) 543-554.

[17] E. Shove, G. Walker, What is energy for? Social practice and energy demand, Theory, Culture \& Society 31 (5) (2014) 41-58.

[18] J. Torriti, A review of time use models of residential electricity demand, Renewable and Sustainable Energy Reviews 37 (2014) 265-272.

[19] J. Torriti, Understanding the timing of energy demand through time use data: Time of the day dependence of social practices, Energy Research \& Social Sciences 25 (2017) 37-47. 
[20] J. Widén, M. Lund, I. Vassileva, E. Dahlquist, K. Ellegärd, E. Wäckelgärd, Constructing load profiles for household electricity and hot water from timeuse data - modelling approach and validation, Energy and Buildings 41 (2009) 753-768.

[21] J. Widén, A. Molin, K. Ellegȧard, Models of domestic occupancy, activities and energy use based on time-use data: deterministic and stochastic approaches with application to various building-related simulations, Building Performance and Simulation 5 (1) (2012) 27-44.

[22] J. L. Ramírez-Mendiola, P. Grünewald, N. Eyre, Linking intra-day variations in residential electricity demand loads to consumers' activities: What's missing?, Energy and Buildings 161 (2018) 63-71.

[23] B. Anderson, Laundry, Energy and Time: Insights from 20 Years of TimeUse Diary Data in the United Kingdom, Energy Research and Social Science 22 (2016) 125-136.

[24] M. Durand-Daubin, B. Anderson, Changing Eating Practices in France and Great Britain: Evidence from Time-Use Data and Implications for Direct Energy Demand, in: Demanding Energy, Palgrave Macmillan, Cham, 2018, pp. 205-231.

[25] P. Grünewald, R. Layberry, Measuring the relationship between time-use and electricity consumption, ECEEE Summer Study Proceedings (2015) 2087-2096.

[26] G. Wood, M. Newborough, Dynamic energy-consumption indicators for domestic appliances: environment, behaviour and design, Energy and Buildings 35 (2003) 821-841.

[27] S. Firth, K. Lomas, A. Wright, R. Wall, Identifying trends in the use of domestic appliances from household electricity consumption measurements, Energy and Buildings 40 (2008) 926-936. 
[28] R. Subbiah, K. Lum, A. Marathe, M. Marathe, Activity based energy demand modeling for residential buildings, in: Innovative Smart Grid Technologies (ISGT), 2013 IEEE PES, IEEE, 2013, pp. 1-6.

[29] E. McKenna, M. Thomson, High-resolution stochastic integrated thermalelectrical domestic demand model, Applied Energy 165 (2016) 445-461.

[30] E. McKenna, S. Higginson, P. Grünewald, S. J. Darby, Simulating residential demand response: Improving socio-technical assumptions in activitybased models of energy demand, Energy Efficiency (2017) 1-15.

[31] M. Durand-Daubin, Household activities through various lenses: crossing surveys, diaries and electric consumption, in: Behavior, Energy and Climate Change Conference (BECC) 2013, UC Berkeley, California, 2013.

[32] P. Kelly, E. Thomas, A. Doherty, T. Harms, Ó. Burke, J. Gershuny, C. Foster, Developing a method to test the validity of 24 hour time use diaries using wearable cameras: a feasibility pilot, PloS one 10 (12) (2015) e0142198.

[33] A. R. Doherty, S. E. Hodges, A. C. King, A. F. Smeaton, E. Berry, C. J. Moulin, S. Lindley, P. Kelly, C. Foster, Wearable cameras in health: The state of the art and future possibilities, American Journal of Preventive Medicine 44 (2013) 320-323.

[34] L. Gemming, A. Doherty, P. Kelly, J. Utter, C. N. Mhurchu, Feasibility of a SenseCam-assisted 24-h recall to reduce under-reporting of energy intake, European Journal of Clinical Nutrition 67 (2013) 1095-1099.

[35] K. C. Armel, A. Gupta, G. Shrimali, A. Albert, Is disaggregation the holy grail of energy efficiency? The case of electricity, Energy Policy 52 (2013) 213-234.

[36] B. Anderson, D. Eyers, R. Ford, D. Giraldo Ocampo, R. Peniamina, J. Stephenson, K. Suomalainen, L. Wilcocks, M. Jack, 
New Zealand GREEN Grid household electricity demand study 20142018doi:10.5255/UKDA-SN-853334.

URL http://reshare.ukdataservice.ac.uk/853334/

[37] P. Kepplinger, G. Huber, J. Petrasch, Field testing of demand side management via autonomous optimal control of a domestic hot water heater, Energy and Buildings 127 (2016) 730-735.

[38] D. Parra, G. S. Walker, M. Gillott, Are batteries the optimum PV-coupled energy storage for dwellings? Techno-economic comparison with hot water tanks in the UK, Energy and Buildings 116 (2016) 614-621.

[39] M. Negnevitsky, K. Wong, Demand-side management evaluation tool, IEEE Transactions on Power Systems 30 (1) (2015) 212-222.

[40] N. Isaacs, M. Camilleri, L. French, Hot water over time - the New Zealand experience, in: XXXV International Association of Housing Science (IAHS) World Congress on Housing Science, BRANZ, 2007.

[41] International Energy Agency, International Energy Agency - Energy Technology Systems Analysis Programme, Technology Brief R03: Water Heating, Tech. rep., International Energy Agency (Jun. 2012).

[42] M. W. Jack, K. Suomalainen, J. J. W. Dew, D. Eyers, A minimal simulation of the electricity demand of a domestic hot water cylinder for smart control, Applied Energy 211 (2018) 104-112.

[43] L. Burrough, K. Saville-Smith, A. Pollard, Heat pumps in New Zealand, Study report SR 329, BRANZ (2015).

[44] M. E. H. Dyson, S. D. Borgeson, M. D. Tabone, D. S. Callaway, Using smart meter data to estimate demand response potential, with application to solar energy integration, Energy Policy 73 (2014) 607-619.

[45] GREEN Grid project, http://www.epecentre.ac.nz/greengrid/, online: accessed 2018-05-23 (2018). 